\title{
Laparoscopic versus vaginal cuff closure in laparoscopic hysterectomy: does it affect female sexuality?
}

\author{
Betul Dundar ${ }^{1}$, Burcu Dincgez Cakmak1, Yeliz Aykanat ${ }^{2}$, Asli Ceren Macunluoglu ${ }^{3}$ \\ ${ }^{1}$ University of Health Sciences, Bursa Yuksek Ihtisas Research and Training Hospital, \\ Department of Obstetrics and Gynecology, Bursa, Turkey \\ ${ }^{2}$ Istanbul Medipol University Faculty of Medicine, Department of Obstetrics and Gynecology, Istanbul, Turkey \\ ${ }^{3}$ Uludag University, Faculty of Medicine, Biostatistics Department, Bursa, Turkey
}

\begin{abstract}
Objectives: To investigate the effect of vaginal cuff closure technique in laparoscopic hysterectomy on vaginal length and female sexual functions.

Material and methods: This study was conducted at a tertiary research hospital. Women who underwent laparoscopic hysterectomy were included and classified according to vaginal cuff closure technique as laparoscopic $(n=75)$ and vaginal route $(n=25)$.Vaginal lengths were measured preoperatively and at 6 th month postoperatively. Golombok-Rust Inventory of Sexual Satisfaction (GRISS) was used to evaluate female sexual functions. SPSS was used for statistical analysis and the level of significance was $p=0.05$.

Results: Preoperative GRISS scores and vaginal lengths were similar in two groups. The shortening of vaginal length and the worsening of GRISS scores were more prominent in vaginal cuff closure group ( $p=0.002, p<0.001)$. The alteration in vaginal length was positively correlated with the alteration in GRISS score in vaginal and laparoscopic route groups $(r=0.800, p<0.001 ; r=0.680, p<0.001)$. The risk of female sexual dysfunction increases 69.88 fold for each $1 \mathrm{~cm}$ shortening of vaginal length $(p=0.039)$. Discriminative value of postoperative vaginal length for female sexual dysfunction in patients who underwent laparoscopic hysterectomy was investigated and a cut-off value of $7.4 \mathrm{~cm}(p<0.001)$ was found. Conclusions: Laparoscopic route instead of vaginal route in laparoscopic hysterectomy is preferable to preserve a better vaginal length, which may be an important factor for female sexual functions.
\end{abstract}

Key words: female sexual function; Golombok-Rust Inventory of Sexual Satisfaction Scale; hysterectomy; laparoscopy; vaginal cuff closure technique

Ginekologia Polska 2019; 90, 7: 365-370

\section{INTRODUCTION}

World Health Organisation has defined sexual health as 'a state of physical, emotional, mental and social well-being in relation to sexuality; not merely the absence of disease, dysfunction or infirmity'. Sex refers to the biological characteristics that define humans as female or male. Women think their uterus as a part of their sexual identity. It is evident that the health problems associated with the reproductive system, including uterus have psychological effects on women. Benign gynecologic diseases such as myoma uteri, abnormal uterine bleeding, endometriosis, pelvic flor diseases often require uterine operations including hysterectomy. In the United States 600,000 women have hysterectomy annually, and $40 \%$ of American women are undergoing this operation until the age of 64 [1].
Hysterectomy has various complications such as bleeding, infections, thromboembolism, genitourinary, gastrointestinal tract and nerve injury, vaginal cuff dehiscence [2]. In addition to these known morbitidies, regardless of the surgical approach its effects on female sexuality and ovarian functions are controversial. Female sexual dysfunction is a multifactorial problem which is defined as a persistent or recurrent disorder of sexual desire, arousal, orgasm and pain. Social conditions such as marital problems, stress, past sexual trauma and medical conditions including diabetes, depression, neurological disorders, alcoholism and drug abuse can affect sexual functions [3]. It was emphasized that age, depression, relationship problems may be factors affecting development of sexual dysfunction after hysterectomy [4]. Dyspareunia due to shortening of vagina as 
a result of removal of cervix, vaginal dryness beginning with pelvic nerve injury and anorgasmic sexual dysfunction resulting from the breakdown of the orgasmic cycle may be underlying mechanisms of posthysterectomy sexual dysfunction [5]. In contrast, there are studies claiming that hysterectomy performed for benign gynecologic diseases may improve female sexual functions [3]. However, there is limited evidence to conclude how the surgical procedure affects sexual function.

Another challenging issue about the effects of hysterectomy on female sexuality is the cuff closure technique. There is only a few data about this issue. In a study searching the effect of horizontal and vertical vaginal cuff closure following vaginal hysterectomy for pelvic organ prolapsus it was demonstrated that there was no difference in female sexuality between two closure techniques [6]. On the other hand, another study investigating the effect of vaginal cuff closure technique after laparoscopic hysterectomy showed improvement in female sexuality through laparoscopic approach [7].

\section{Objectives}

We aimed to investigate the effect of vaginal cuff closure technique used in laparoscopic hysterectomy on female sexuality. The secondary aim of this study was to determine a cut-off value for vaginal length to cause detoriation in female sexuality.

\section{MATERIAL AND METHODS}

A cross sectional study was conducted at a university affiliated tertiary research and training hospital in Northwestern region of Turkey among 100 women, who underwent laparoscopic hysterectomy for benign uterine pathologies between January 2016-January 2017. Women between 35-55 years old who planned to undergo laparoscopic hysterectomy for benign uterine pathologies were included in the study. Patients $(n=100)$ were preoperatively assessed and further classified according to the vaginal cuff closure technique as laparoscopic closure $(n=75)$ and vaginal closure $(n=25)$. The exclusion criteria of the present study were as follows; 1) Current or past psychiatric diseases, 2) Psychiatric drug use, 3) Patients with malignancy and/or endometriosis, 4) Presence of uterin prolapses at any degrees 5) History of any other gynecologic operations, 6) Presence of chronic debilitating disease, 7) Drug abuse, 8) Failure to attend the follow-up visit. All patients underwent gynecologic examination including pap-smear testing and sonographic examination. The type of surgery was determined according to medical conditions, informed consent and shared decision making. Baseline demographic characteristics including obstetric and gynecologic history such as gravida, parity, first age of coit, menarche age, education level, income level, delivery mode, age, height, weight, body mass index (BMI), surgical and medical history were recorded. Vaginal length was measured preoperatively and at $6^{\text {th }}$ month postoperatively by the surgical team. Operations were performed by the same surgical technique. Vaginal length was measured from the hymenal ring to the $D$ point of POP-Q by speculum examination preoperatively on the day of hospitalization and postoperatively at $6^{\text {th }}$ month during the routine gynecology outpatient clinic control by the same operator. Care was taken not to stretch the vagina during the measurement.

All the patients included in the present study underwent conventional laparoscopic hysterectomy. At vaginal cuff closure step of the laparoscopic hysterectomy for both vaginal and laparoscopic approaches the vaginal cuff was formed by suturing the vaginal opening antero-posteriorly in a horizontal pattern. The first bite of the suture was merged with the uterosacral ligament at one side and vaginal opening was closed through a continous running suture until the last bite of the suture which also merges with the uterosacral ligament on the contralateral side. The suture material used in either laparoscopic or vaginal closure methods was 0-Vicryl. The surgeries were done in the same hospital and by the same surgical team accepting the same surgical techniques for the conventional laparascopic hysterectomy.

Differences between the two groups in terms of sexual dysfunction were evaluated using the validated Turkish version of the Golombok-Rust Inventory of Sexual Satisfaction (GRISS) scale. Patients were asked to complete GRISS at preoperative and postoperative periods. When patients were admitted to hospital for preoperative evaluation by anesthesia care team 2 weeks before the surgery they were asked to complete the GRISS. At $6^{\text {th }}$ month postoperatively, when patients admitted to gynecology outpatient clinic for routine postoperative evaluation, they were asked to complete the GRISS for one more time. The questionnaires were given to the patients by an obstetrics and gynecology assistant and they were asked to answer the questionnaires alone in a room by themselves.

GRISS has 28 questions all of which are answered over five points. It is Likert type scale and answers are as follows: always, usually, sometimes, hardly ever and never. It evaluates the different domains of sexual function which can be sorted as infrequency, non-communication, female dissatisfaction, vaginismus, female non-sensuality, female avoidence and anorgasmia. The GRISS is evaluated with a total scale score and for subdomains. High GRISS scores are indicative of more severe sexual dysfunction and disruption in relationship quality. Raw scores are converted to standard scores between 1 and 9 . Scores $\geq 5$ are indicative of detoriation in sexual functions. An increase in the scores for each subdomain also shows impaired sexual function $[8,9]$. Differences between pre and postoperative $6^{\text {th }}$ months GRISS scores were calculated and given as alteration in 
GRISS scores. The alterations and preoperative GRISS scores were compared between two groups.

A post hoc power analysis was conducted using a large effect size, based upon findings of the present study. Large effect size was obtained by comparing mean alterations in vaginal length which were calculated from laparoscopic closure $(1.51 \pm 0.59)$ and vaginal closure $(2.02 \pm 0.68)$ terms for 100 participants. Using this effect size $(d=0.80)$ with a sample size of 100 participants, achieved power was estimated as $93 \%$ at the significance level of $a=0.05$. Shapiro Wilk test was used for assessing whether the variables follow normal distribution. Continuous variables were presented as median (minimum:maximum) and mean \pm standard deviation where categorical variables were reported as $n$ (\%). According to normality test independent $t$ test and Mann Whitney U tests were used for group comparisons. Pearson chi-square and Fisher-Freeman-Halton tests were used for comparing categorical variables. Correlations between discrete and continuous variables were analyzed using Spearman correlation analysis and correlation coefficient was calculated. For postoperative vaginal length, receiver operator curve (ROC) analysis was performed for the cut-off point that could predict sexual dysfunction. Also, the sensitivity and specificity of postoperative vaginal length for predicting sexual dysfunction were calculated by ROC analysis. Area under curve (AUC) value with 95\% confidence interval (CI) were reported. To determine independent risk factors that affect sexual function, logistic regression analysis was performed. SPSS version 20 (IBM Corp. Released 2012.IBM SPSS Statistics for Windows, Armonk, NY:IBM Corp.) was used for performing statistical analysis and $p \leq 0.05$ was considered statistically significant.

The study was approved by the local ethics committee and written informed consent was obtained from each participant. Also, it complies with the Declaration of Helsinki.

\section{RESULTS}

The sociodemographic findings of patients were demonstrated in Table 1. There was no difference between two groups according to age, height, weight, BMI, gravida, parity, first age of coit, menarche, delivery mode, education and income level ( $p>0.05)$.

Preoperative vaginal length, preoperative GRISS score, alteration in GRISS score and in vaginal length were compared between two groups (Tab. 2). Although there was no difference in regard of preoperative GRISS score and vaginal length, the shortening of vaginal length was more prominent in vaginal closure group $(p=0.002)$ and also, the worsening in GRISS score was more apparent in vaginal group $(p<0.001)$

The data about the alterations in vaginal length and GRISS score at $6^{\text {th }}$ month were analyzed to show whether

Table 1. Sociodemographic characteristics of patients

\begin{tabular}{|c|c|c|c|}
\hline & $\begin{array}{l}\text { Laparoscopic Closure } \\
(n=75)\end{array}$ & $\begin{array}{l}\text { Vaginal Closure } \\
(n=25)\end{array}$ & $\mathbf{p}$ \\
\hline Age [y] & $47.41 \pm 4.11$ & $47.24 \pm 4.28$ & $0.857^{\mathrm{a}}$ \\
\hline Height $[\mathrm{cm}]$ & $164(155: 172)$ & $165(155: 170)$ & $0.051^{b}$ \\
\hline Weight [kg] & $70.36 \pm 10.52$ & $73.68 \pm 7.97$ & $0.152^{\mathrm{a}}$ \\
\hline Body mass index $\left[\mathrm{kg} / \mathrm{m}^{2}\right]$ & $26.27 \pm 3.58$ & $26.99 \pm 2.83$ & $0.308^{a}$ \\
\hline Gravida [n] & $3(1: 6)$ & $3(1: 5)$ & $0.454^{b}$ \\
\hline Parity [n] & $3(1: 6)$ & $2(1: 5)$ & $0.455^{b}$ \\
\hline First age of coit [y] & $25(16: 34)$ & $25(17: 29)$ & $0.722^{b}$ \\
\hline Menarche age [y] & $13(11: 16)$ & $13(11: 16)$ & $0.240^{b}$ \\
\hline $\begin{array}{l}\text { Education level } \\
\text { - Literate }(n, \%) \\
\text { - Primary school }(n, \%) \\
\text { - Secondary school }(n, \%) \\
\text { - High school }(n, \%) \\
\text { - University }(n, \%)\end{array}$ & $\begin{array}{l}6(8) \\
18(24) \\
17(22.7) \\
24(32) \\
10(13.3)\end{array}$ & $\begin{array}{l}3(12) \\
5(20) \\
6(24) \\
8(32) \\
3(12)\end{array}$ & $0.976^{c}$ \\
\hline $\begin{array}{l}\text { Income level } \\
\text { - Low }(n, \%) \\
\text { - Moderate }(n, \%) \\
\text { - High }(n, \%)\end{array}$ & $\begin{array}{l}30(40) \\
32(42.7) \\
13(17.3)\end{array}$ & $\begin{array}{l}8(32) \\
11(44) \\
6(24)\end{array}$ & $0.688^{c}$ \\
\hline $\begin{array}{l}\text { Delivery mode } \\
\text { - Cesarean section }(n, \%) \\
\text { - Normal delivery }(n, \%) \\
\text { - Cesarean and normal delivery }(n, \%)\end{array}$ & $\begin{array}{l}22(29.3) \\
31(41.4) \\
22(29.3)\end{array}$ & $\begin{array}{l}6(24) \\
12(48) \\
7(28)\end{array}$ & $0.820^{d}$ \\
\hline
\end{tabular}

${ }^{a}$ — Independent sample $t$ test; ${ }^{b}$ — Mann-Whitney-U Test; ${ }^{c}$ — Fisher Freeman Halton Test; ${ }^{d}$ — Chi-square Test 
Table 2. Vaginal length and GRISS scores of patients

\begin{tabular}{|l|l|l|l|}
\hline & $\begin{array}{l}\text { Laparoscopic Closure } \\
(\mathbf{n = 7 5 )}\end{array}$ & $\begin{array}{l}\text { Vaginal Closure } \\
(\mathbf{n = 2 5 )}\end{array}$ & $\mathbf{p}$ \\
\hline Preoperative vaginal length [cm] & $9.60 \pm 0.54$ & $9.52 \pm 0.69$ & $0.952^{\mathrm{a}}$ \\
\hline Alterations in vaginal length [cm] & $1.51 \pm 0.59$ & $2.02 \pm 0.68$ & $\mathbf{0 . 0 0 2}^{\mathrm{a}}$ \\
\hline Preoperative total GRISS score & $5.59 \pm 1.82$ & $4.88 \pm 2.15$ & $0.076^{\mathrm{a}}$ \\
\hline Alteration in GRISS score & $1.29 \pm 0.98$ & $3.28 \pm 1.49$ & $\mathbf{0 . 0 0 1}^{\mathrm{a}}$ \\
\hline
\end{tabular}

a - Mann Whitney U Test

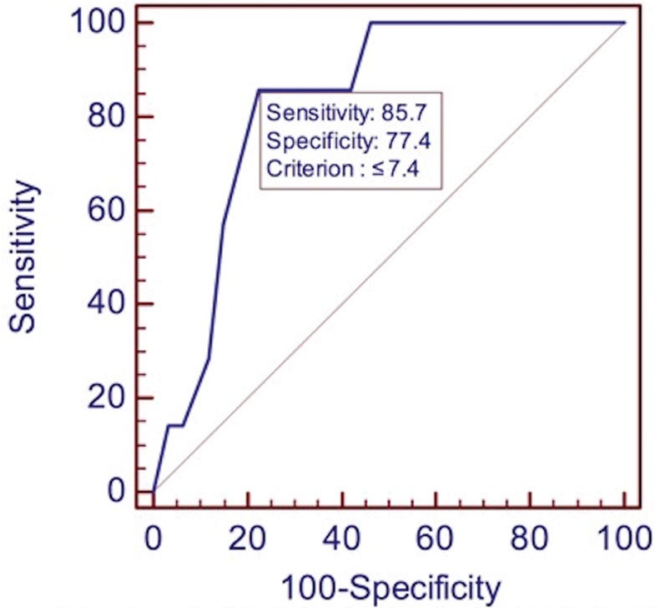

Figure 1. Receiver operating curve performed for determining the cut-off point of postoperative vaginal length that could predict sexual dysfunction

there is a correlation between the shortening of vagina and increased GRISS scores. We found that the alteration in vaginal length was positively correlated with the alteration in GRISS score for all patients $(r=0.756, p<0.001)$ and also for both vaginal closure $(r=0.800, p<0.001)$ and laparoscopic closure $(r=0.680, p<0.001)$ groups. Furthermore, logistic regression analysis revealed that the risk of female sexual dysfunction increased 69.88 fold for each $1 \mathrm{~cm}$ shortening in vaginal length $(p=0.039)$.

The discriminative value of postoperative vaginal length for sexual dysfunction in patients who underwent laparoscopic hysterectomy was evaluated by ROC curve. AUC for postoperative vaginal length was 0.829 with a sensitivity of $85.7 \%$ and specificity of $77.4 \%$ with a cut-off value of $7.4 \mathrm{~cm}$ $(p<0.001)$ (Fig. 1).

\section{DISCUSSION}

Main findings of the present study are as follows: 1 ) the shortening of vaginal length and the worsening in GRISS score were more prominent in vaginal cuff closure group, 2) the shortening in vaginal length was positively correlated with the increase in GRISS scores for all patients and also for both vaginal cuff closure and laparoscopic cuff closure groups, 3) the risk of female sexual dysfunction was found to be increased 69.88 fold for each $1 \mathrm{~cm}$ shortening in vaginal length, 4) postoperative vaginal length with a cut-off value of $7.4 \mathrm{~cm}$ was supposed to be discriminative for female sexual dysfunction.

Female sexuality is a challenging issue with several confounding factors some of which are medical, psychological, physical, social, educational, religious and hormonal [10].

A majority of gynecologic surgeries have been thought to be closely related with female sexual dysfunctions. Among those, hysterectomy, which is the most frequently performed gynecological operation, has been under investigation for its effects on female sexuality for a quite long time. However, it is still controversial whether female sexual functions improve or worsen following hysterectomy [11]. Improvement in female sexual functions after hysterectomy were reported to be associated with relief of symptoms such as dysmenorrhea, dyspareunia and uterine bleeding, all of which may result in an improved quality of life including sexual functions [12]. On the other hand, neurovascular injuries due to surgical procedure, ovarian failure due to postoperative diminished blood flow, simultaneously performed bilateral salphingo-oophorectomy and decreased vaginal length following operation may provide an explanation for the worsening of sexual functions following hysterectomy [13]. Furthermore, uterus is an organ that has a psychosocial importance and so, hysterectomy may refer to loss of sexuality for most of the women [10].

It is obvious that the decisions of surgeon about the operation will have a postoperative consequence. Not only the surgical approach to hysterectomy but also the surgical technique chosen will affect surgical results. Therefore, when to prefer which surgical method should be questioned for several aspects [14].

The term laparoscopic hysterectomy defines application of laparoscopic technique for at least a part of operation [15]. It was first introduced by Reich et al in 1989 [16]. The advantages and disadvantages have been discussed since that time. However, nowadays the number of hysterectomies performed through laparoscopic approach is quite high and increasing gradually [14].

There are studies in the literature investigating the effects of laparoscopic hysterectomy on female sexuality and 
comparing the results with that of abdominal and vaginal hysterectomies. The study, in which vaginal, abdominal and laparoscopic hysterectomies were compared in regard of their effects on female sexuality, revealed no differences between three groups according to their effects on orgasm, frequency and desire [10]. Sexual functions were shown to be improved in postoperative third month and second year when patients who underwent vaginal hysterectomy and total abdominal hysterectomy were compared. However, patients in total abdominal hysterectomy group experienced more pain and their self-image were poorer [17]. The impact of abdominal and laparoscopic hysterectomies on female sexuality was analyzed in a study by using Arizona Sexual Experiences Scale. It is observed that sexual functions were improved after both types of hysterectomies and total laparoscopic hysterectomy were shown to have significantly better outcomes for sexual drive and arousal [18]. A study comparing postoperative prevalence of hypoactive sexual desire disorder after different types of hysterectomy including abdominal, vaginal, laparoscopic assisted vaginal, laparoscopic supracervical and total laparoscopic hysterectomies did not display any differences between these five surgical approaches. On the other hand, female sexual function scores were reported to be better among patients in laparoscopic supracervical and total laparoscopic hysterectomy groups [19]. Probably, due to the smaller size of abdominal scar and the shorter recovery time after surgeries, laparoscopic methods were shown to be associated with positive effects on females' self-esteem and quality of life. Less invasive surgical methods of hysterectomy such as vaginal and laparoscopic routes tend to have less destruction on female sexuality [18]. From this point of view, it is possible to infer that even the different surgical techniques used during any surgical approach may have different effects on surgical outcomes [14]. For instance; total versus subtotal hysterectomy, different techniques used to support vaginal vault, to or not to perform bilateral oophorectomy during hysterectomy under elective circumstances, to or not to use several techniques in laparoscopic hysterectomies which may be helpful in avoiding several complications such as using vaginal delineators, rectal probes or illuminated ureteric stents will have different surgical outcomes [14].

How vaginal length is affected following different types of gynecologic procedures especially hysterectomies is not a widely studied topic. Pelvic reconstructive surgery and hysterectomy, regardless of type, were shown to be determinants of vaginal length with a shortening effect [20]. When vaginal length following total abdominal and vaginal hysterectomies were compared a significantly shorter vaginal length was observed after vaginal hysterectomy [21]. De La Cruz et al. compared 38 total vaginal hysterectomies and 46 robotic hysterectomies, both of which were accompanied by pelvic support surgery, with regard to vaginal length and postoperative sexual functions. Although vaginal lengths were stated to be decreased after total vaginal hysterectomy as compared to robotic hysterectomy, any differences were not observed between two groups according to the sexual functions [22].

The vaginal lengths of sexually active women were observed to be longer when compared to sexually inactive women. However, the difference between two groups was not significant. Generally, vaginal length and caliber were not accepted as determinants of sexual activity and sexual function scores were not shown to be affected by vaginal size [23]. Yet, it is important to refer that vaginal length may be an indicator of sexual functions in case of presence of a positive history for gynecologic surgery [24]. Total vaginal length was affected following total abdominal, total laparoscopic and vaginal hysterectomies. Each type of hysterectomy was shown to decrease vaginal length similarly and female sexual functions were disturbed irrespective of the surgical approach [25].

Bastu et al [7] randomized patients who underwent laparoscopic hysterectomy for the route of vaginal cuff closure where either laparoscopic or vaginal route was preferred. They reported that although female sexual functions did not differ significantly pre and three months postoperatively, vaginal lengths were significantly longer in laparoscopic route group.

In this study, the vaginal length was shown to be decreased significantly in vaginal cuff closure group. Similarly to the other studies in the literature, we evaluated the correlation between vaginal length and female sexual dysfunction and we demonstrated a positive correlation between the shortening in vaginal length and the increase in GRISS scores for all patients who underwent laparoscopic hysterectomy and also separately for vaginal cuff closure and laparoscopic cuff closure groups. In the previous studies with similar study design as ours, only postoperative female sexual functions and postoperative vaginal lengths were evaluated and the correlation analysis was performed with regard to those two parameters. However, dissimilarly, in the correlation analysis we take into account the difference between the pre and postoperative vaginal lengths and the GRISS scores, which might be more informative about the degree of vaginal shortening and the deterioration in female sexual functions, instead of taking only postoperative vaginal lengths and postoperative GRISS scores. This provide us the chance of demonstrating risk of female sexual dysfunction increased nearly 70 fold for each $1 \mathrm{~cm}$ shortening in vaginal length. Moreover, we found that to avoid postoperative female sexual dysfunction a vaginal length of minimum $7.4 \mathrm{~cm}$ was required.

There are few limitations of this study. First of all, this is a non randomized study from a single center with a small 
sample size. Secondly, 6 months is a short time period for reevaluation of patients postoperatively. Thirdly, there were difficulties in measuring the vaginal length since vagina is a quite elastic organ. Moreover, pain and afraid associated with postoperative period are subjective components of sexual limitation and therefore it is difficult to differentiate their effects on sexual functions. Lastly, the surgical factors in vaginal cuff closure group and in laparoscopic cuff closure group, such as the duration of operation, blood loss, the time of passing flatus, the time of postoperative mobilization and the postoperative complications were not considered in this study.

\section{CONCLUSIONS}

In conclusion, patients should be informed about that the laparoscopic hysterectomy may cause deterioration in sexual functions to various extent. Considering the correlation of vaginal length with GRISS scores it should be better to avoid shortening of vagina as much as possible. Therefore, laparoscopic horizontal closure of the vaginal cuff instead of vaginal horizontal closure is preferable to maintain a better vaginal length which can be an important factor for female sexual functions.

\section{Conflict of interests}

The authors report no conflict of interests.

\section{REFERENCES}

1. Centers for Disease Control and Prevention. Women's reproductive health: hysterectomy. Centers for Disease Control and Prevention. Women's reproductive health: hysterectomy. Available online at: http://www.cdc.gov/reproductivehealth/womensrh/hysterectomy. htm. (28.01.2013).

2. Clarke-Pearson DL, Geller EJ. Complications of hysterectomy. Obstet Gynecol. 2013; 121(3): 654-673, doi: 10.1097/AOG.0b013e3182841594, indexed in Pubmed: 23635631.

3. Lonnée-Hoffmann R, Pinas I. Effects of Hysterectomy on Sexual Function. Curr Sex Health Rep. 2014; 6(4): 244-251, doi: 10.1007/s11930-014-00293, indexed in Pubmed: 25999801.

4. Laumann EO, Paik A, Rosen RC. Sexual dysfunction in the United States: prevalence and predictors. JAMA. 1999; 281(6): 537-544, doi: 10.1001/jama.281.6.537, indexed in Pubmed: 10022110.

5. Ramdhan RC, Loukas M, Tubbs RS. Anatomical complications of hysterectomy: A review. Clin Anat. 2017; 30(7): 946-952, doi: 10.1002/ca.22962, indexed in Pubmed: 28762535

6. Uçar MG, Illhan TT, Şanlıkan F, et al. Sexual functioning before and after vaginal hysterectomy to treat pelvic organ prolapse and the effects of vaginal cuff closure techniques: a prospective randomised study. Eur J Obstet Gynecol Reprod Biol. 2016; 206: 1-5, doi: 10.1016/j. ejogrb.2016.08.041, indexed in Pubmed: 27612212.

7. Bastu E, Yasa C, Dural O, et al. Comparison of 2 Methods of Vaginal Cuff Closure at Laparoscopic Hysterectomy and Their Effect on Female Sexual Function and Vaginal Length: A Randomized Clinical Study. J Minim Invasive Gynecol. 2016; 23(6): 986-993, doi: 10.1016/j.jmig.2016.07.007, indexed in Pubmed: 27426680.
8. Rust J, Golombok S, Rust J, et al. The Golombok-Rust Inventory of Sexual Satisfaction (GRISS). Br J Clin Psychol. 1985; 24 ( Pt 1)(2): 63-64, indexed in Pubmed: 3971070.

9. Tuğrul C, Öztan N, Kabakçı E. Golombok-Rust Cinsel Doyum Ölçeği'nin standardizasyon çalışması. [The validation study of Golombok-Rust Inventory of sexual satisfaction]. Türk Psikiyatri Dergisi. 1993; 4: 83-88.

10. Ayoubi JM, Fanchin R, Monrozies X, et al. Respective consequences of abdominal, vaginal, and laparoscopic hysterectomies on women's sexuality. Eur J Obstet Gynecol Reprod Biol. 2003; 111(2): 179-182, indexed in Pubmed: 14597248.

11. LEE J, CHOI J, HONG J, et al. Does conventional or single port laparoscopically assisted vaginal hysterectomy affect female sexual function? Acta Obstetricia et Gynecologica Scandinavica. 2011; 90 (12): 1410-1415, doi: 10.1111/j.1600-0412.2011.01255.x.

12. Rhodes JC, Kjerulff KH, Langenberg PW, et al. Hysterectomy and sexual functioning. JAMA. 1999; 282(20): 1934-1941, doi: 10.1001/jama.282.20.1934, indexed in Pubmed: 10580459.

13. Abdelmonem AM. Vaginal length and incidence of dyspareunia after total abdominal versus vaginal hysterectomy. Eur J Obstet Gynecol Reprod Biol. 2010; 151(2): 190-192, doi: 10.1016/j.ejogrb.2010.03.031, indexed in Pubmed: 20427116.

14. Johnson N, Barlow D, Lethaby A, et al. Surgical approach to hysterectomy for benign gynaecological disease. Cochrane Database Syst Rev. 2005(1): CD003677, doi: 10.1002/14651858.CD003677.pub2, indexed in Pubmed: 15674911.

15. Garry R, Fountain J, Mason Su, et al. The eVALuate study: two parallel randomised trials, one comparing laparoscopic with abdominal hysterectomy, the other comparing laparoscopic with vaginal hysterectomy. BMJ. 2004; 328(7432): 129, doi: 10.1136/bmj.37984.623889.F6, indexed in Pubmed: 14711749.

16. Reich H, DeCaprio J, McGlynn F. Laparoscopic Hysterectomy. J Gynecol Surg. 1989; 5(2): 213-216, doi: 10.1089/gyn.1989.5.213.

17. Gütl $P$, Greimel ER, Roth $R$, et al. Women's sexual behavior, body image and satisfaction with surgical outcomes after hysterectomy: a comparison of vaginal and abdominal surgery. J Psychosom Obstet Gynaecol. 2002; 23(1): 51-59, indexed in Pubmed: 12061038.

18. Kürek Eken $M$, İlhan $G$, Temizkan $O$, et al. The impact of abdominal and laparoscopic hysterectomies on women's sexuality and psychological condition. Turk J Obstet Gynecol. 2016; 13(4): 196-202, doi: 10.4274/tjod.71245, indexed in Pubmed: 28913121.

19. Lermann J, Häberle L, Merk S, et al. Comparison of prevalence of hypoac tive sexual desire disorder (HSDD) in women after five different hysterectomy procedures. Eur J Obstet Gynecol Reprod Biol. 2013; 167(2): 210-214, doi: 10.1016/j.ejogrb.2012.12.005, indexed in Pubmed: 23313224.

20. Tan JS, Lukacz ES, Menefee SA, et al. Determinants of vaginal length Am J Obstet Gynecol. 2006; 195(6): 1846-1850, doi: 10.1016/j. ajog.2006.06.063, indexed in Pubmed: 17014819.

21. Chen $B$, Ren DP, Li JX, et al. Comparison of vaginal and abdominal hysterectomy:A prospective non-randomized trial. Pak J Med Sci. 2014; 30(4): 875-879, indexed in Pubmed: 25097536.

22. De La Cruz JF, Myers EM, Geller EJ. Vaginal versus robotic hysterectomy and concomitant pelvic support surgery: a comparison of postoperative vaginal length and sexual function. J Minim Invasive Gynecol. 2014; 21(6): 1010-1014, doi: 10.1016/j.jmig.2014.04.011, indexed in Pubmed: 24780383.

23. Schimpf MO, Harvie HS, Omotosho TB, et al. Society of Gynecologic Surgeons Fellows' Pelvic Research Network. Does vaginal size impact sexual activity and function? Int Urogynecol J. 2010; 21(4): 447-452, doi: 10.1007/s00192-009-1051-2, indexed in Pubmed: 19960183.

24. Celik H, Gurates B, Yavuz A, et al. The effect of hysterectomy and bilaterally salpingo-oophorectomy on sexual function in post-menopausal women. Maturitas. 2008; 61(4): 358-363, doi: 10.1016/j.maturitas.2008.09.015, indexed in Pubmed: 18977621.

25. Ercan Ö, Özer A, Köstü B, et al. Comparison of postoperative vaginal length and sexual function after abdominal, vaginal, and laparoscopic hysterectomy. Int J Gynaecol Obstet. 2016; 132(1): 39-41, doi: 10.1016/j. ijgo.2015.07.006, indexed in Pubmed: 26475076. 